\title{
First-in-Human Randomized Trial to Assess Safety, Tolerability, Pharmacokinetics and Pharmacodynamics of the KDM1A Inhibitor Vafidemstat
}

\author{
Rosa María Antonijoan ${ }^{1,2}$. Juan Manuel Ferrero-Cafiero ${ }^{1}$. Jimena Coimbra ${ }^{1}$. Montse Puntes ${ }^{1}$. \\ Joan Martínez-Colomer ${ }^{1} \cdot$ María Isabel Arévalo $^{3} \cdot$ Cristina Mascaró $^{3} \cdot$ Cesar Molinero $^{3} \cdot$ Carlos Buesa $^{3}$. \\ Tamara Maes ${ }^{3}$ (])
}

Accepted: 12 February 2021 / Published online: 23 March 2021

(c) The Author(s) 2021

\begin{abstract}
Background Vafidemstat, an inhibitor of the histone lysine-specific demethylase KDM1A, corrects cognition deficits and behavior alterations in rodent models. Here, we report the results from the first-in-human trial of vafidemstat in healthy young and older adult volunteers. A total of 110 volunteers participated: 87 were treated with vafidemstat and 23 with placebo.

Objectives The study aimed to determine the safety and tolerability of vafidemstat, to characterize its pharmacokinetic and pharmacodynamic profiles, to assess its central nervous system (CNS) exposure, and to acquire the necessary data to select the appropriate doses for long-term treatment of patients with CNS disease in phase II trials.

Methods This single-center, randomized, double-blind, placebo-controlled phase I trial included a single and 5-day repeated dose-escalation and open-label CNS penetration substudy. Primary outcomes were safety and tolerability; secondary outcomes included analysis of the pharmacokinetics and pharmacodynamics, including chemoprobe-based immune analysis of KDM1 A target engagement (TE) in peripheral blood mononuclear cells (PBMCs) and platelet monoamine oxidase B (MAOB) inhibition. CNS and cognitive function were also evaluated.

Results No severe adverse events (AEs) were reported in the dose-escalation stage. AEs were reported at all dose levels; none were dose dependent, and no significant differences were observed between active treatment and placebo. Biochemistry, urinalysis, vital signs, electrocardiogram, and hematology did not change significantly with dose escalation, with the exception of a transient reduction of platelet counts in an extra dose level incorporated for that purpose. Vafidemstat exhibits rapid oral absorption, approximate dose-proportional exposures, and moderate systemic accumulation after 5 days of treatment. The cerebrospinal fluid-to-plasma unbound ratio demonstrated CNS penetration. Vafidemstat bound KDM1A in PBMCs in a dose-dependent manner. No MAOB inhibition was detected. Vafidemstat did not affect the CNS or cognitive function. Conclusions Vafidemstat displayed good safety and tolerability. This phase I trial confirmed KDM1A TE and CNS penetration and permitted characterization of platelet dynamics and selection of phase IIa doses.
\end{abstract}

Trial registration EUDRACT No. 2015-003721-33, filed 30 October 2015.

Tamara Maes

tmaes@ oryzon.com

1 Centre d'Investigació del Medicament, Institut de Recerca de l'Hospital de la Santa Creu i Sant Pau (IIB-Sant Pau), Barcelona, Spain

2 Pharmacology and Therapeutics Department, Universitat Autònoma de Barcelona (UAB), Bellaterra, Spain

3 Oryzon Genomics S.A. Carrer Sant Ferran 74, Cornellà de Llobregat, 08940 Barcelona, Spain

\section{Key Points}

Vafidemstat displays good safety and tolerability in the dose range $0.2-2.5 \mathrm{mg} /$ day.

A chemoprobe-based assay revealed that vafidemstat effectively bound the KDM1A target in humans.

Vafidemstat displays central nervous system penetration. 


\section{Introduction}

The etiology of sporadic neurodegenerative disorders such as Alzheimer's or Parkinson's disease is currently unknown. Epigenetics plays an important role in brain development and the establishment and loss of memory and cognition [1-4]; modulation of epigenetic factors may present a unique opportunity for treatment of central nervous system (CNS) diseases [5].

$\mathrm{KDM} 1 \mathrm{~A}$ is a histone demethylase that has been proposed as a target for the treatment of neurodegenerative or neurodevelopmental diseases [6-8].

Vafidemstat (ORY-2001) is an orally bioavailable and brain penetrant compound that was originally identified as a dual inhibitor of the flavin adenine dinucleotide (FAD)dependent amine oxidases KDM1 A (EC 1.14.99.66) and monoamine oxidase B (MAOB; EC 1.4.3.4) [10]. KDM1A is a histone demethylase that acts in the nucleus and is involved in transcription regulation [9]. MAOB is structurally related but involved in dopamine metabolism and is a validated drug target in Parkinson's disease.

In vitro, vafidemstat inhibits the catalytic activity of KDM1A and MAOB through irreversible binding to their FAD cofactors. Vafidemstat also interferes with the capacity of KDM1A to interact with part of its recruiting transcription factors. In vivo, it primarily inhibits KDM1A, and MAOB inhibition is not significant in the therapeutic dose range. Vafidemstat restored cognition and behavior alterations in several rodent models [10]. Comparison of vafidemstat with selective KDM1A or MAOB inhibitors revealed that KDM1A inhibition is key to the efficacy of vafidemstat. In the brain, KDM1A is recruited by transcription factors, including serum response factor, and is involved in behavior alterations; vafidemstat promotes the response of immediate early genes [10]. Vafidemstat also has anti-inflammatory activity in the CNS, evidenced by its efficacy in mouse models for multiple sclerosis (MS) [11]. In rodents, the therapeutic effects of vafidemstat can be achieved in the absence of an impact on hematopoiesis, a known on-target effect of KDM1A inhibition [12, 13].

Here, we report the results of a first-in-human phase I clinical trial in healthy young and older adult volunteers. The study aimed to determine the safety and tolerability of vafidemstat, to characterize its pharmacokinetic and pharmacodynamic parameters, and to assess its CNS exposure. The data obtained from this study were used to select the appropriate doses for longterm treatment of patients with CNS disease in phase II trials.

\section{Materials and Methods}

\subsection{Study Drug and Treatment}

The investigational medicinal drug substance and drug product (placebo or vafidemstat $0.2,1,1.5$, or $4 \mathrm{mg}$ in hydroxypropyl methylcellulose capsules) were manufactured in accordance with good manufacturing practices (GMP) guidelines and labeled according to Annex 13 of the EU GMP guidelines. The chemical structure of the compound is shown in Fig. 1a. The maximum recommended starting dose (MRSD) was $0.2 \mathrm{mg}$, as calculated in the most sensitive species (rat). The dose-escalation regimens were 0.2, 0.6, $1.5,2.5$, and $4 \mathrm{mg}$ for the single ascending dose (SAD) study and $0.2,0.6,1,1.5,2.5 \mathrm{mg}$, and an additional dose level of $4.0 \mathrm{mg}$ for the multiple ascending dose (MAD) study. In the cerebrospinal fluid (CSF) substudy, the doses were 2 and 4 mg (Fig. 1c, d). Blinding, randomization, and dose-escalation procedures are available in Supplemental Materials and Methods in Online Resource.

\subsection{Study Design}

This study was designed as a single-center, randomized, double-blind, placebo-controlled phase I trial to assess the safety, tolerability, pharmacokinetics, and pharmacodynamics of vafidemstat. It consisted of a SAD study in male volunteers, a MAD study in healthy young and older adult populations, and a substudy of CSF.

The primary objectives of the study were to assess the safety and tolerability of vafidemstat; the secondary objectives were to assess the pharmacokinetic and pharmacodynamic profiles of vafidemstat after (1) single ascending oral dose administration in healthy young male volunteers, (2) repeated (5 days) ascending daily oral dose administration in young male and female healthy subjects, and (3) repeated (5 days) daily oral dose administration in older adults.

The MRSD was determined according to US FDA and European Medicines Agency guidelines [14, 15] based on available preclinical data. The no observed adverse effect level (NOAEL) in nonclinical good laboratory practices

Fig. 1 Clinical trial design. a Chemical structure of vafidemstat. b Impact of vafidemstat on platelet levels in nonclinical species, represented as \% inhibition compared with control animals. All values are expressed as $\mathrm{mg} / \mathrm{m}^{2} /$ day to allow for direct comparison. Differences in administration (once daily by oral gavage in rats and dogs vs. drinking water in mice) were accounted for. Doses in $\mathrm{mg} / \mathrm{kg} /$ day were converted to $\mathrm{mg} / \mathrm{m}^{2} /$ day by multiplication with the $\mathrm{Km}$ factor for each species (3, 6, 20, 37 for mouse, rat, dog, human, respectively). Black triangles indicate the SAMP8 efficacy studies in mouse; gray circles indicate the 28-day toxicity in rats; white squares indicate the 28-day toxicity in dogs; black arrows represent the doses chosen for the SAD and MAD cohorts; gray arrows represent those for the CSF cohorts in the phase I trial. The maximum recommended starting dose was $0.2 \mathrm{mg}$. c Clinical trial doses per cohort. d Study design of the SAD, $\mathrm{MAD}$, and CSF cohorts. Dark gray rectangle indicates the sentinel subject, light gray rectangles indicate subjects in active treatment; white rectangles indicate subjects receiving placebo. CSF cerebrospinal fluid, $M A D$ multiple ascending dose, p.o. oral administration, PLT platelet, $Q D$ once daily, $S A D$ single ascending dose 
a<smiles>Nc1nnc(CNC2C[C@H]2c2ccc(OCc3ccccc3)cc2)o1</smiles>

b

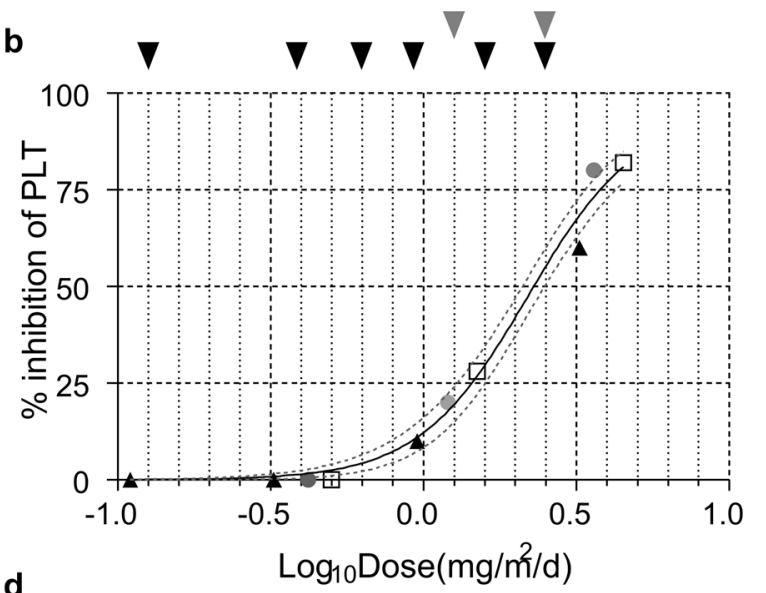

d

\begin{tabular}{|ccccc|}
\hline $\begin{array}{c}\text { Cohort } \\
\text { SAD }\end{array}$ & $\begin{array}{c}\text { Cohort } \\
\text { MAD }\end{array}$ & $\begin{array}{c}\text { Cohort } \\
\text { CSF }\end{array}$ & $\begin{array}{c}\text { Dose } \\
(\mathrm{mg} / \mathrm{d})\end{array}$ & $\begin{array}{c}\mathrm{Log}_{11} \text { dose } \\
\left(\mathrm{mg} / \mathrm{m}^{2} / \mathrm{d}\right)\end{array}$ \\
\hline I & I & - & 0.2 & -0.90 \\
\hline II & II & - & 0.6 & -0.43 \\
\hline- & III & - & 1.0 & -0.20 \\
\hline III & IV & - & 1.5 & -0.03 \\
\hline- & - & I & 2.0 & 0.10 \\
\hline IV & V & - & 2.5 & 0.19 \\
\hline V & VI & II & 4.0 & 0.40 \\
\hline
\end{tabular}
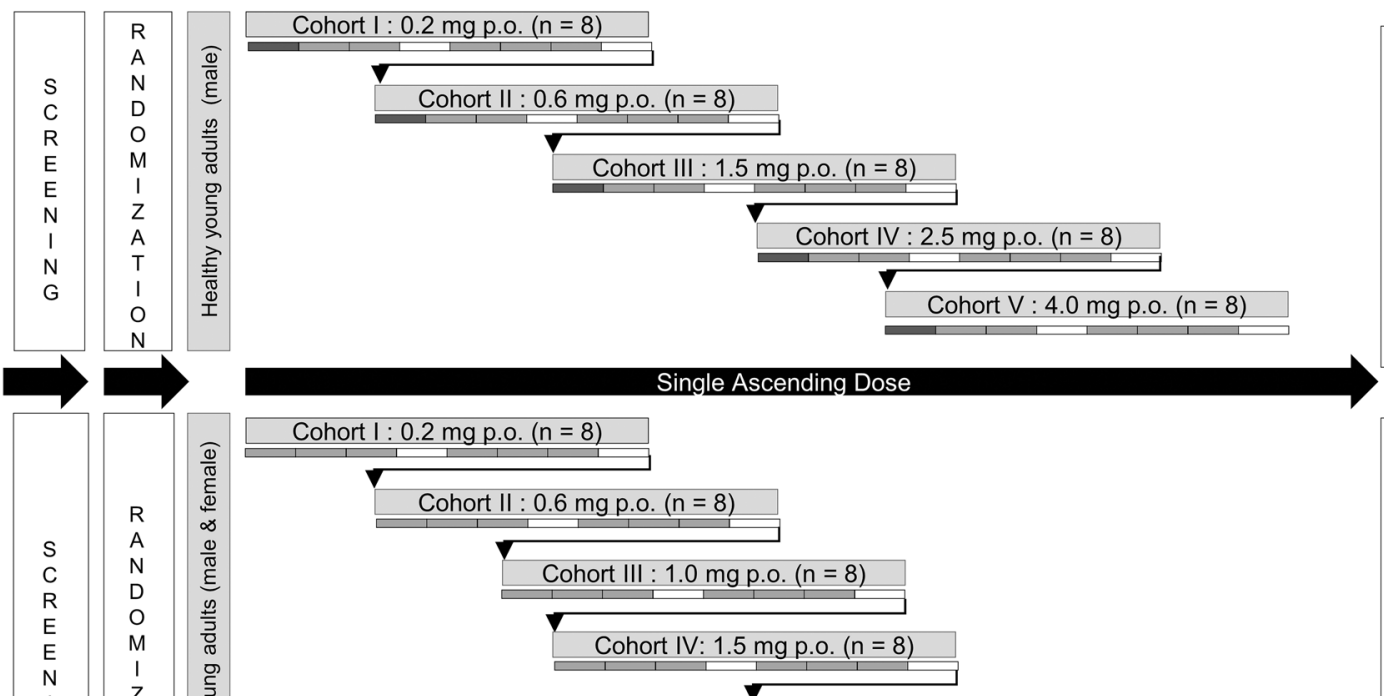

Cohort V: 2.5 mg p.o. $(n=8)$

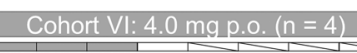

Cohort V: $2.5 \mathrm{mg}$ p.o. $(n=4)$

Multiple Ascending Dose (QD, 5ON/2OFF)

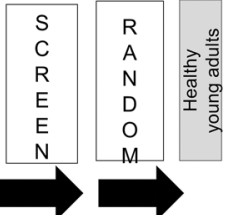

CSF Cohort I: $2.0 \mathrm{mg}$ p.o. $(\mathrm{n}=9)$

CSF Cohort II: $4.0 \mathrm{mg}$ p.o. $(\mathrm{n}=9)$ 
(GLP) toxicology studies in rats and dogs was established at 0.200 and $0.075 \mathrm{mg} / \mathrm{kg}$, respectively. The MRSD was calculated based on the NOAEL in a 28-day GLP toxicity study in rats, the most sensitive species:

MRSD $=1 /$ safety factor $\times \mathrm{Km}$, rat $/ \mathrm{Km}$, human $\times$ NOAELrat $\times$ human body weight $=0.2 \mathrm{mg}$, where the safety factor $=10 ; \mathrm{Km}$, rat $=6 ; \mathrm{Km}$, human $=37 ;$ NOAELrat $=$ $0.2 \mathrm{mg} / \mathrm{kg}$; and human body weight $=60 \mathrm{~kg}$.

The dose escalation was designed to commence from the MRSD and continue to the minimum intolerable dose (MID). The MID was defined as the dose at which a serious adverse event (SAE) occurs in one or more subjects and the causality is established as certainly, probably, or possibly related to the study drug; when $>50 \%$ of subjects experience a limiting grade 2 or higher AE after active treatment but not placebo; when $>50 \%$ of AEs occur in subjects under active treatment but not placebo; or (in view of the role of KDM1 A in hematopoiesis) when a $>50 \%$ decrease in platelet levels from baseline or grade 1 or higher thrombocytopenia platelet values are found in at least $>50 \%$ of subjects under active treatment but not placebo.

The original dose escalation regimens designed were 0.2 , $0.6,1.5,2.5$, and $4 \mathrm{mg}$ for the SAD and 0.2, 0.6, 1, 1.5, and $2.5 \mathrm{mg}$ for the MAD. This dose range was based on the nonclinical toxicology and efficacy data, was equivalent to the therapeutic dose in preclinical species, and was designed to identify the onset of hematological engagement (Fig. 1b). An extra cohort at $4 \mathrm{mg}$ was incorporated into the MAD study after protocol modification. In the CSF pharmacokinetic substudy, the doses were 2 and $4 \mathrm{mg}$ (Fig. 1d).

The eligibility criteria and programmed interventions are presented in Tables S1 and Tables S2-S4 in ESM-2, respectively.

\subsection{Study Population and Assessments}

Healthy adult male (SAD) and male or female subjects (MAD) complying with the eligibility criteria as shown in Table S1 in ESM-2 were randomized and included in the study and dosed in the SAD, MAD, and CSF studies. Vafidemstat or placebo were administered as a single oral dose (SAD and CSF pharmacokinetics) or as a daily oral dose for 5 consecutive days (MAD).

Physical examination included vital signs, AE checklist, electrocardiograph (ECG), and neurological evaluations: visual analogue scale (VAS), Leeds Sleep Evaluation Questionnaire (LSEQ), Wisconsin Card Sorting Test (WCST), and Sternberg test. Blood samples were obtained for safety laboratory tests (hematology, biochemistry, and urinalysis) and at baseline and preestablished timepoints (see Tables S2-S4 in ESM-2) after the first drug administration for pharmacokinetic analysis using a GMP-validated method and for pharmacodynamic analysis to assess KDM1A target engagement (TE) and platelet MAOB activity in the SAD and MAD studies. An open-label CSF pharmacokinetics study was included to assess the pharmacokinetic profile in the CNS after a single oral dose of vafidemstat. Details about the assessments, analytical methods, and statistics are available in ESM-1.

\subsection{Analytical Methods}

\subsubsection{Pharmacokinetics}

Plasma and CSF samples for pharmacokinetic analysis were obtained predose and at preestablished timepoints. Vafidemstat concentrations were determined in human plasma and CSF using a highly sensitive GLP-validated liquid chromatography-tandem mass spectrometry method (lower limit of quantification $10 \mathrm{pg} / \mathrm{mL}$ for plasma and $50 \mathrm{pg} / \mathrm{mL}$ for CSF) with electron spray ionization in positive ion mode and deuterated vafidemstat as internal standard, developed at Laboratorios Echevarne, S.A, Spain. Liquid-liquid extraction with ethyl acetate was used for sample processing, and chromatography was run on a Venusil XBP C18 column, with $0.1 \%$ formic acid in water and acetonitrile as mobile phases A and B, respectively. Intra- and interday precision in the validation ranged between 0.57 and $9.75 \%$ for plasma and between 1.18 and $5.63 \%$ for CSF. Intra- and interday accuracy (bias) in the validation ranged between -4.74 and $9.50 \%$ of the nominal values for plasma and between -8.39 and $10.25 \%$ of the nominal values for CSF. Incurred sample reanalysis was conducted on study samples to confirm the reproducibility of the bioanalytic method. All the repeat and original samples $(n=244$ distributed into six independent bioanalytical batches for plasma and $n=10$ into two batches for CSF) were within $20 \%$ of the mean of the two values, meeting the preestablished acceptance criteria of $67 \%$ and therefore confirming the reproducibility of the assay. Sample analysis was GLP compliant, and pharmacokinetic parameters-including the dose normalized maximum (peak) concentration $\left(C_{\max }\right)$, time to reach $C_{\max }\left(t_{\max }\right)$, and area under the plasma concentration-time curve (AUC)-were calculated by means of noncompartmental analysis using WinNonlin ${ }^{\circledR} 2.1$ software. Plasma-concentration-versus-time profiles are presented on linear or log-linear scale mean profiles \pm standard error of the mean for each treatment.

\subsubsection{Plasma Unbound Concentrations}

Plasma unbound concentrations were calculated by correcting the measured total concentrations with the plasma fraction unbound value of 0.029 , which had been separately assessed in vitro by equilibrium dialysis using the HTDialysis ${ }^{\circledR}$ system at Advinus Therapeutics Ltd., India. 


\subsubsection{KDM1A Target Engagement}

For the evaluation of KDM1A TE (\% of KDM1 bound to vafidemstat), a proprietary chemoprobe-based assay $[16,17]$ was used at Oryzon Genomics S.A., Spain. Briefly, blood samples were collected in EDTA (ethylenediaminetetraacetic acid) K2 tubes from healthy volunteers at baseline and prespecified timepoints. Peripheral blood mononuclear cells (PBMCs) were obtained by density gradient centrifugation in LeucoSep ${ }^{\mathrm{TM}}$ tubes. Cells were lysed in $1 \mathrm{X}$ cell lysis buffer (Cell Signaling; \#9803) containing 1X Complete Mini, protease inhibitor cocktail tablets (ROCHE; \# 11836153001) in the presence of $25 \mathrm{nM}$ biotinylated KDM1A chemoprobe OG-881. The chemoprobe binds the free KDM1A (i.e., not occupied by vafidemstat) in the sample. This lysate was then used to perform a chemiluminescent KDM1A sandwich enzyme-linked immunosorbent assay (ELISA) (using antiKDM1A-coated ELISA plates) and a chemoprobe ELISA (using streptavidin-coated ELISA plates) to determine both total and free KDM1A in the sample. Streptavidin (Promega Biotech Ibérica; \#Z7041) at $10 \mu \mathrm{g} / \mathrm{mL}$ in phosphate buffered saline (PBS) or mouse monoclonal anti-KDM1A antibody (Abcam; \#ab53269) at $2 \mu \mathrm{g} / \mathrm{mL}$ in PBS were used to coat LumiNunc Plates MaxiSorp (NUNC; \#436110) plates. Rabbit monoclonal anti-KDM1A antibody (Cell Signaling; \#2184) at $0.125 \mu \mathrm{g} / \mathrm{mL}$ in PBS was used for primary detection, and the peroxidase-conjugated secondary antirabbit immunoglobulin $\mathrm{G}$ antibody (Jackson ImmunoResearch; \#711-035-152) at 1:5000 dilution in PBS was used for secondary detection in combination with $100 \mu \mathrm{L} /$ well of chemiluminescent substrate (Invitrogen; \#37074). Antibody \#2184 recognizes the N-terminal and \#ab53269 the $\mathrm{C}$-terminal region of KDM1A in the native complex. The KDM1 A antibodies used were KDM1 A knockout validated, and we confirmed that coincubation with synthetic peptides corresponding to the respective target epitopes eliminated the KDM1A signal. Intraday precision of the validation in human PBMC ranged between 0.31 and $5.08 \%$ for total KDM1A and between 1.03 and $8.02 \%$ for free KDM1A. Interday precision was 11.9 and $19.7 \%$ for total and free KDM1A, respectively.

All ELISA and chemoprobe ELISA assays were run as technical triplicates. After raw data processing (robust elimination of outliers according to Grubbs criteria and subtraction of blank signal), the TE or percentage of KDM1A bound by vafidemstat was calculated relative to the predose sample $\left(T_{0}\right)$ from the same subject as follows:
$\operatorname{TEx}(\%)=100-100 \times \operatorname{Rx}(\%) / \operatorname{RTo}(\%)$,

where

$R(\%)=\left(\frac{\text { RLUfree }}{\text { RLUtotal }}\right) \times 100$.

\section{Results}

The first subject was enrolled on 4 April 2016, and the last subject completed the study on 2 June 2017 . Subjects were screened during the 28 days prior to dosing.

A total of 40 healthy young male subjects were enrolled in the SAD study and treated with placebo or vafidemstat (randomization ratio 2:6) at doses ranging from 0.2 to $4 \mathrm{mg}$. Eight subjects were enrolled per dose level.

In the MAD study, 40 healthy young male and female subjects were enrolled and treated for 5 days with placebo or vafidemstat (randomization ratio 2:6) at doses ranging from 0.2 to $2.5 \mathrm{mg}$ per day. Eight subjects were enrolled per each of the dose levels. After a protocol modification, four additional young subjects were enrolled in a sixth cohort and treated for 5 days with placebo or vafidemstat (randomization ratio 1:3) at $4 \mathrm{mg}$ per day. Finally, four older adult subjects were enrolled and treated for 5 days with placebo or vafidemstat at $2.5 \mathrm{mg}$ per day (randomization ratio 1:3).

The demographics and baseline characteristics of the subjects are summarized in Table S5 in ESM-2. In the SAD and MAD studies, the distribution of demographics was similar in the treated and placebo arms. All subjects were Caucasian.

\subsection{Safety and Tolerability}

A total of 120 AEs were reported during the three study stages (SAD, MAD, and older adult population): 90 AEs by subjects receiving vafidemstat and 30 AEs by subjects receiving placebo, i.e., the same proportion as the treatment to placebo randomization (3:1). Of these, 108 AEs were classified as treatment-emergent AEs (TEAEs) possibly or probably related to the study drug: 79 TEAEs were reported in volunteers treated with vafidemstat and 29 in volunteers treated with placebo. No differences in the proportion of subjects reporting AEs or differences between the AEs reported were observed between the active treatment and placebo. No SAEs were reported during the study.

During the SAD stage in healthy young male volunteers $(N=40), 15$ AEs were reported; 13 were considered to be treatment related (12 in subjects receiving vafidemstat and one in the placebo arm). Nine subjects experienced at least one $\mathrm{AE}$. In the vafidemstat treatment group, the most 
common AEs were headache, followed by euphoric mood. None of the AEs were dose dependent.

During the MAD stage in healthy young male/female volunteers $(N=44)$, a total of 69 AEs were reported (51 AEs with vafidemstat and 18 with placebo). The most common AEs were headache, followed by dry mouth, asthenia, postural dizziness, and somnolence. In total, 61 AEs were related to the study treatment (44 with vafidemstat and 17 with placebo). The data from a subcohort $(N=4)$ subject to a dosing error are represented separately in Table S6 in ESM-2. None of the AEs were considered severe. Moderate AEs (all headaches experienced with vafidemstat) were reported in dose levels I $(0.2 \mathrm{mg} /$ day $)$, III $(1.0 \mathrm{mg} /$ day $)$, and IV $(1.5 \mathrm{mg} /$ day) but not in the highest dose levels (V and VI, 2.5 and $4 \mathrm{mg} /$ day, respectively). The rest of the AEs were considered mild. Regarding the AEs reported in the highest dose levels, the proportion of subjects who experienced the most common AEs was similar in the active treatment and placebo arms.

Safety and tolerability results from the MAD stage in young male and female volunteers supported the initiation of the MAD stage in older adult volunteers at dose level VI ( $4.0 \mathrm{mg} /$ day, $N=4$; three active and one placebo). A total of 23 AEs were reported: 12 with vafidemstat and 11 with placebo. Three subjects reported at least one AE. The most common AEs were postural dizziness (in a subject in the placebo arm) followed by headache, blunted affect, and vertigo. Most reported AEs were mild. A total of 21 treatmentrelated TEAEs were reported (10 with vafidemstat and 11 with placebo). TEAEs possibly or probably related to the study drug in the SAD, MAD, and older adult cohort are presented in Table 1.

No significant changes were observed over time in hematological, biochemical, or urinalysis parameters or in vital signs or ECG values in the planned SAD or MAD (including older adult) cohorts. Platelet levels showed no variations at any of the SAD dose levels or up to MAD dose III $(1.0 \mathrm{mg} /$ day). At MAD dose levels IV (1.5 mg/day) and V (2.5 mg/ day), platelet counts were within the normal range, but a tendency for lower counts was emerging in the active treatment arm compared with the placebo arm. At 1.5 and $2.5 \mathrm{mg} /$ day, a slight reduction $(-11$ and $-21 \%$ ) of the mean was observed by $192 \mathrm{~h}$ after the first administration and a small rebound ( +15 and $+18 \%$ ) was observed in the follow-up visit (Fig. 2a, b). Although the differences between placebo and active treatment arms were not significant, these changes were thought to reflect the first indication of hematopoietic engagement. A protocol amendment was issued to incorporate an additional MAD dose (level VI; $N=4$; placebo or $4 \mathrm{mg} /$ day) to characterize the hematopoietic impact. At this dose level, a transient reduction of platelet levels was observed by $192 \mathrm{~h}$, and platelet evolution was monitored to characterize the nadir and rebound. Platelets dropped below
$50 \%$ of baseline levels in two of three vafidemstat-treated subjects by $216 \mathrm{~h}$ after the first treatment administration and in all subjects at $240 \mathrm{~h}$ and $264 \mathrm{~h}$ and recovered during the follow-up visits (Fig. 2c). Based on these data, the MID as defined per protocol was met at dose $4 \mathrm{mg} / \mathrm{day}$, and the maximum tolerated dose was established at $2.5 \mathrm{mg}$ /day.

In summary, no SAEs or AEs leading to treatment discontinuation were reported in the SAD or MAD cohorts. With exception of the effect on platelets observed in the extra cohort (4 mg/day) as described, all hematology, biochemistry, and urinalysis parameters, as well as vital signs and ECG assessments in SAD and MAD, were within normal ranges in the vafidemstat treatment arms and revealed no significant differences between treatment arms.

\subsection{Pharmacokinetics}

In the SAD and MAD cohorts, the plasma pharmacokinetic profiles of vafidemstat after single (Fig. 3a) and multiple (Fig. 3b) dosing reflected rapid oral absorption, with a $t_{\max }$ of $\sim 0.5-2 \mathrm{~h}$, moderate variability (overall coefficient of variation $\leq 30 \%)$, a relatively long half-life $(\sim 20-30 \mathrm{~h})$, and approximately dose-proportional exposures (based on dose normalized $C_{\max }$ and AUC). A tendency for more than proportional increases was found starting from the dose of $2.5 \mathrm{mg} /$ day in the MAD cohort, which could result from a saturation of drug absorption and/or elimination at higher dose levels, among other causes. Apparent clearance was low $(6 \mathrm{~L} / \mathrm{h})$. A moderate systemic accumulation (mean accumulation ratios $\left[\mathrm{AUC}_{0-t \text { Day } 5} / \mathrm{AUC}_{0-\mathrm{t} \text { Day } 1}\right] \sim 2$ ) was observed after 5 days of administration, and pharmacokinetic modeling indicated that a stationary state would be reached between approximately days 5 and 7 (data not shown). Pharmacokinetic profiles at $2.5 \mathrm{mg}$ /day did not differ significantly between the young and older adult populations (Fig. 3b). The mean apparent volume of distribution was $150 \mathrm{~L}$, indicating potentially low to moderate distribution to tissues (Table 2).

In the CSF cohort, vafidemstat was shown to cross the human blood-brain barrier, with quantifiable concentrations found from $2 \mathrm{~h}$ post-dose, the first timepoint analyzed. Additionally, $\mathrm{CSF} /$ plasma (unbound) ratios observed in this study (mean of 0.81) suggested that no or minor CNS-toblood efflux transport of vafidemstat is expected in humans (Fig. 3c and Table 2).

\subsection{Pharmacodynamics}

Failure to efficiently inhibit the target is a factor that contributes to the low success rate of clinical drug development and underscores the relevance of demonstrating target occupancy in early-stage clinical trials. To evaluate the pharmacodynamics of vafidemstat, we used a chemoprobe-based 
Table 1 Number of TEAEs (and percentage of total TEAEs) possibly or probably related to the study drug by preferred term and dose levels/( $n$ $=$ subjects) in the SAD, MAD, and older adult cohorts

\begin{tabular}{|c|c|c|c|c|c|c|c|}
\hline $\begin{array}{l}\text { Cohort and MedDRA AE term } \\
\text { SAD cohort }\end{array}$ & $\begin{array}{l}\text { Placebo } \\
n=10\end{array}$ & $\begin{array}{l}\mathrm{I}(0.2 \mathrm{mg} / \mathrm{day}) \\
n=6\end{array}$ & $\begin{array}{l}\text { II }(0.6 \mathrm{mg} / \mathrm{day}) \\
n=6\end{array}$ & $\begin{array}{l}\text { III ( } 1.0 \mathrm{mg} / \text { day }) \\
n=0\end{array}$ & $\begin{array}{l}\text { III ( } 1.5 \mathrm{mg} / \text { day }) \\
n=6\end{array}$ & $\begin{array}{l}\text { IV ( } 2.5 \mathrm{mg} / \text { day }) \\
n=6\end{array}$ & $\begin{array}{l}\mathrm{V}(4.0 \mathrm{mg} / \text { day }) \\
n=6\end{array}$ \\
\hline Diarrhea & $1(7.7)$ & & & & & & \\
\hline Headache & & $1(7.7)$ & $1(7.7)$ & & & & $1(7.7)$ \\
\hline Feeling hot & & & $1(7.7)$ & & & & \\
\hline Euphoric mood & & & $1(7.7)$ & & & & $1(7.7)$ \\
\hline Musculoskeletal stiffness & & & & & $1(7.7)$ & & \\
\hline Paresthesia & & & & & $1(7.7)$ & & \\
\hline Abdominal discomfort & & & & & & $1(7.7)$ & \\
\hline Dry mouth & & & & & & $1(7.7)$ & \\
\hline Asthenia & & & & & & $1(7.7)$ & \\
\hline Blunted affect & & & & & & & $1(7.7)$ \\
\hline Total $13(100)$ & $1(7.7)$ & $1(7.7)$ & $3(23.1)$ & & $2(15.4)$ & $3(23.1)$ & $3(23.1)$ \\
\hline MAD cohort & $n=11$ & $n=6$ & $n=6$ & $n=6$ & $n=6$ & $n=6$ & $n=3$ \\
\hline Palpitations & & $1(1.6)$ & & & & & \\
\hline Dry mouth & $5(8.2)$ & & & $4(6.5)$ & $3(4.9)$ & $1(1.6)$ & \\
\hline Asthenia & $2(3.3)$ & $2(3.3)$ & $1(1.6)$ & $1(1.6)$ & & & \\
\hline Feeling hot & $1(1.6)$ & $1(1.6)$ & & & & & \\
\hline Muscle spasms & & $1(1.6)$ & & & & & \\
\hline Dizziness postural & $2(3.3)$ & $1(1.6)$ & & $1(1.6)$ & & & \\
\hline Headache & $3(4.9)$ & $3(4.9)$ & $1(1.6)$ & $2(3.3)$ & $2(3.3)$ & & $2(3.3)$ \\
\hline Dyspepsia & & & $1(1.6)$ & & & & \\
\hline Salivary hypersecretion & $1(1.6)$ & & & & $1(1.6)$ & & \\
\hline Feeling drunk & & & $1(1.6)$ & & & & \\
\hline Somnolence & & & $2(3.3)$ & & $1(1.6)$ & & \\
\hline Apathy & & & $1(1.6)$ & & & & \\
\hline Blunted affect & & & $2(3.3)$ & & & & \\
\hline Euphoric mood & & & $1(1.6)$ & & & & \\
\hline Daydreaming & & & $1(1.6)$ & & & & \\
\hline Diplopia & $1(1.6)$ & & & & & & \\
\hline Vision blurred & $1(1.6)$ & & & & & & \\
\hline Constipation & $1(1.6)$ & & & $1(1.6)$ & & & \\
\hline Feeling cold & & & & $3(4.9)$ & & & \\
\hline Nasopharyngitis & & & & $1(1.6)$ & & & \\
\hline Hematoma & & $1(1.6)$ & & & & & \\
\hline Total $61(100)$ & $17(27.9)$ & $10(16.4)$ & $11(18.0)$ & $13(21.3)$ & $7(11.5)$ & $1(1.6)$ & $2(3.3)$ \\
\hline Elderly cohort & $n=1$ & & & & & $n=3$ & \\
\hline Palpitations & & & & & & $1(4.8)$ & \\
\hline Vertigo & $1(4.8)$ & & & & & $1(4.8)$ & \\
\hline Diarrhea & $2(9.5)$ & & & & & & \\
\hline Asthenia & & & & & & $1(4.8)$ & \\
\hline Feeling cold & & & & & & $1(4.8)$ & \\
\hline Gait disturbance & & & & & & $1(4.8)$ & \\
\hline Musculoskeletal stiffness & & & & & & $1(4.8)$ & \\
\hline Postural dizziness & $4(19.0)$ & & & & & & \\
\hline Headache & & & & & & $3(14.3)$ & \\
\hline Somnolence & $1(4.8)$ & & & & & & \\
\hline Blunted effect & $2(9.5)$ & & & & & $1(4.8)$ & \\
\hline Euphoric mood & $1(4.8)$ & & & & & & \\
\hline Total $21(100)$ & $11(52.4)$ & & & & & $10(47.6)$ & \\
\hline
\end{tabular}

Data are presented as $\mathrm{n}(\%)$. AEs occurring on the same day are only counted once

$A E$ adverse event, $M A D$ multiple ascending dose, MedDRA Medical Dictionary for Regulatory Activities, SAD single ascending dose, TEAE treatment-emergent adverse event 
Fig. 2 Hematological impact of vafidemstat. Impact of vafidemstat on platelet levels at a $1.5 \mathrm{mg} /$ day, b $2.5 \mathrm{mg} /$ day, and (c) the extra dose level of $4 \mathrm{mg} /$ day, represented as $\%$ change from baseline levels. Black dots indicate vafidemstat (volunteers analyzed $n=6$ in $[\mathbf{a} / \mathbf{b}]$ or $n=$ 3 in [c]); open circles indicate placebo treatment (volunteers analyzed $n=2$ in [a/b] or $n=$ 1 in [c]); black arrows represent dosing occasions. Data are presented as means and standard error of the mean. $C F B$ change from baseline a

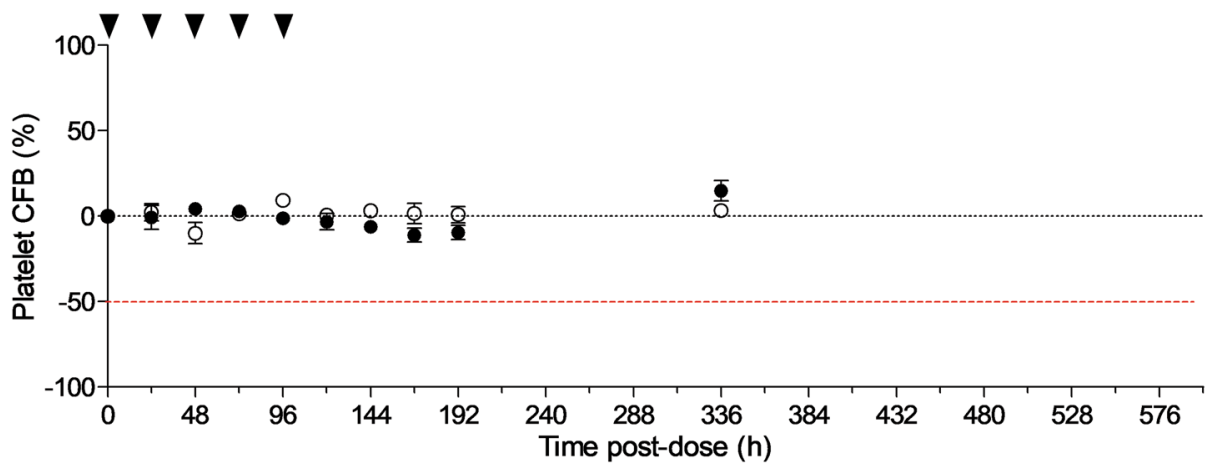

b

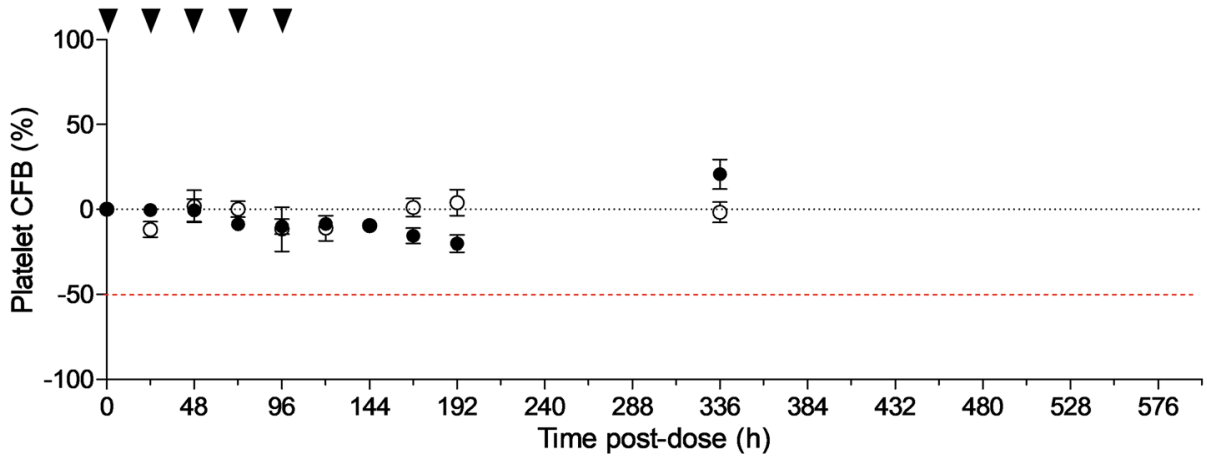

C

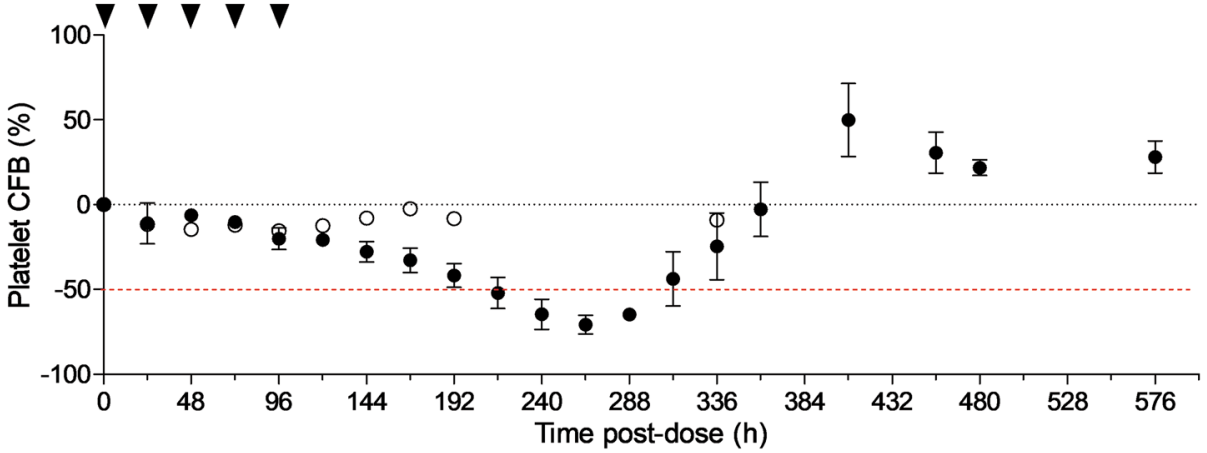

immunoassay $[16,17]$. The assay was validated for use in clinical trials and used to calculate the TE from the amount of free (uninhibited) and total KDM1A protein in PBMC samples obtained predose and at different timepoints after administration (Fig. 4a, b). Vafidemstat bound KDM1A in PBMCs in a dose-dependent manner. In the SAD cohorts, a maximum KDM1A TE of around $80 \%$ was observed at $12 \mathrm{~h}$ (4 mg), and substantial binding remained $72 \mathrm{~h}$ after dosing (Fig. 4c). KDM1A TE values observed $12 \mathrm{~h}$ after the last dose in the MAD study (108 h after first dose) were similar to those found in the SAD study. Maximum TE values of up to $90 \%$ were reached at $108 \mathrm{~h}$. KDM1A TE turnover was slow, with 40-60\% TE remaining at $192 \mathrm{~h}$ (96 h after last dose, Fig. 4d). KDM1A TE increased with plasma exposure, reaching a peak of $\sim 90 \%$ at $C_{\text {max,ss }}$ (where ss is steady state) of $\sim 30 \mathrm{ng} / \mathrm{mL}$ and AUCss of $\sim 500 \mathrm{ng} \cdot \mathrm{h} / \mathrm{mL}$. Thus, a clear relationship was evidenced between the $C_{\max } / \mathrm{AUC}$ parameters and the amount of vafidemstat bound to KDM1 A in the tested dose range (Fig. 4e, f). Similarly, the impact on platelets increased with exposure, reaching level drops (from baseline) of $\sim 50 \%$ at $C_{\text {max,ss }}$ of $\sim 100 \mathrm{ng} / \mathrm{mL}$ and $\mathrm{AUC}_{\mathrm{ss}}$ of $1000 \mathrm{ng} \cdot \mathrm{h} / \mathrm{mL}$ (Fig. 4g, h). KDM1A TE was $85.6 \%$ in the older adult population (evaluable sample from a single individual), comparable with that observed in young volunteers at the same dose level. As far as we can tell, this is the first time a chemoprobe-based method has been used to effectively monitor the dynamics of TE in a clinical trial.

KDM1A TE in the brain could not be tested in this study because no KDM1A positron emission tomography tracer was available. However, since vafidemstat binds KDM1A in the human brain and PBMC samples ex vivo with comparable efficacy (data not shown), and the calculated plasma 
a
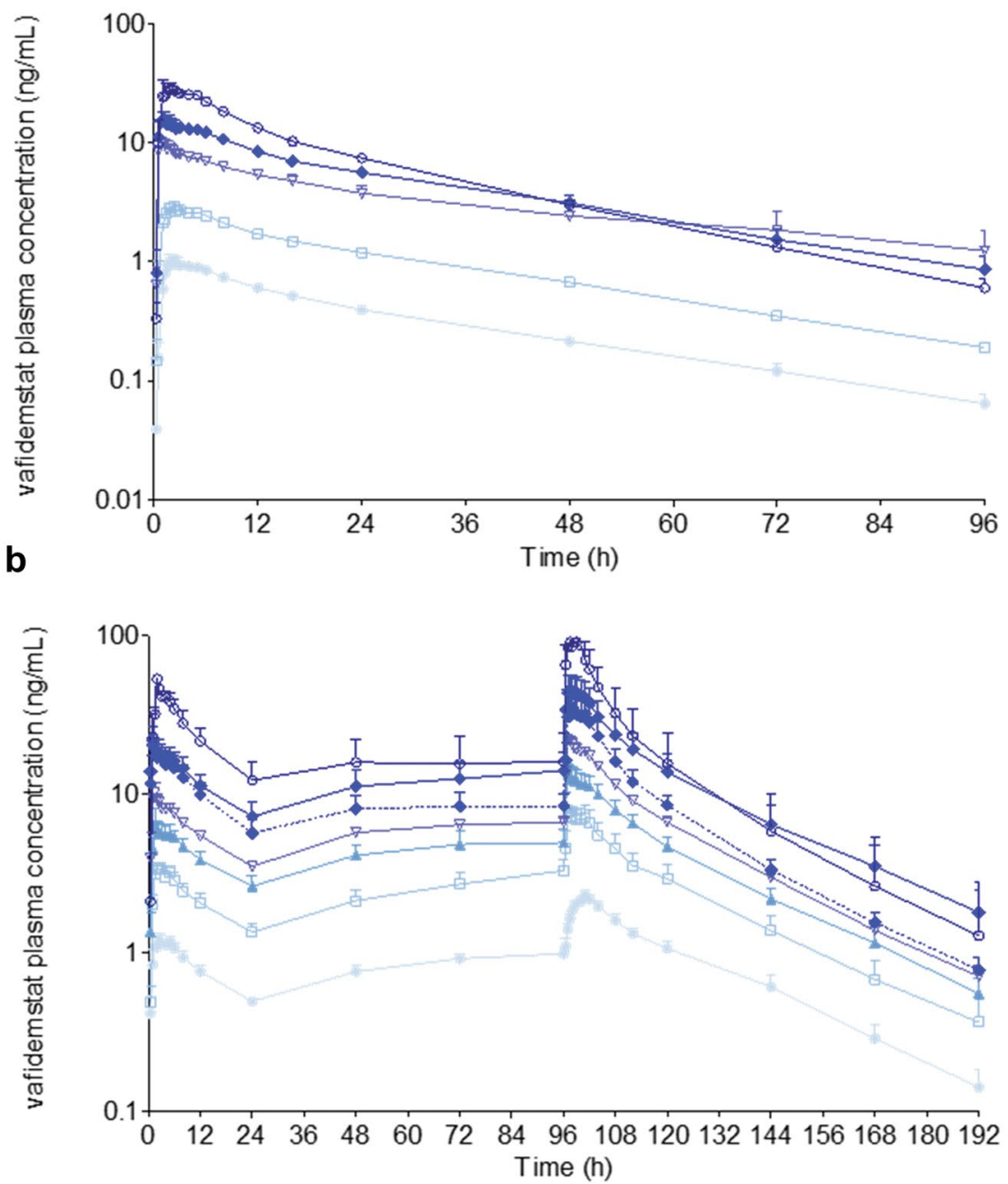

C

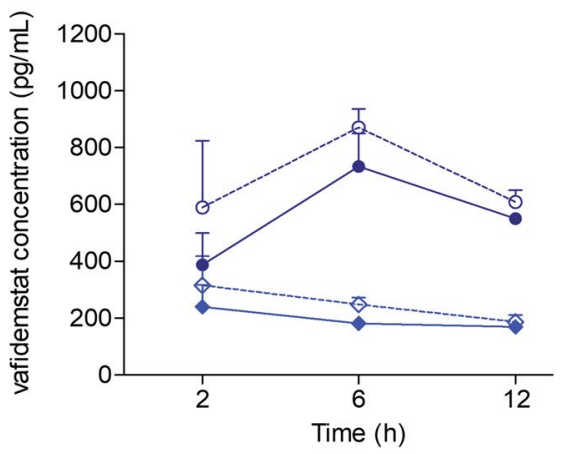

Fig. 3 Vafidemstat pharmacokinetics. (a) Plasma concentrations after single and (b) multiple ascending doses in healthy young (full lines) and older adult (dotted line) subjects. Light blue circles $0.2 \mathrm{mg}$; white squares $0.6 \mathrm{mg}$; light blue triangles $1 \mathrm{mg}$; white triangles 1.5 $\mathrm{mg}$; blue diamonds $2.5 \mathrm{mg}$; white circles $4 \mathrm{mg}$. Data are presented as mean and standard error of the mean. Volunteers analyzed at each datapoint, $\mathrm{n}=6$ except for MAD $4 \mathrm{mg}, \mathrm{n}=3$. (c) Cerebrospinal fluid (full lines) and plasma (unbound, dotted lines) concentrations after a single dose of $2 \mathrm{mg}$ (diamonds) or $4 \mathrm{mg}$ (circles)

cohorts, as assessed using a VAS. Subjects reported feeling "partially calm" throughout the study, a feeling shared by those receiving placebo or vafidemstat regardless of the dose level administered and attributed to participation in the study. Vafidemstat did not disrupt sleeping or awakening patterns, as assessed by the LSEQ in the SAD, MAD, or CSF cohorts. A few spurious individual VAS values composing the global score reached statistical significance, but no dose response was observed. No significant differences were observed at any dose level or parameter in the WCST or Sternberg test in the SAD, MAD, or CSF cohorts.

or reveal a potential for abuse in the SAD, MAD, or CSF 
Table 2 Pharmacokinetics and central nervous system penetrance of vafidemstat

\begin{tabular}{|c|c|c|c|c|c|c|c|}
\hline \multirow{2}{*}{$\begin{array}{l}\text { Cohort and param- } \\
\text { eter } \\
\text { SAD }^{\mathrm{a}}\end{array}$} & \multicolumn{7}{|c|}{ Dose level and results } \\
\hline & $\begin{array}{l}\text { I: } 0.2 \mathrm{mg} \\
(n=6)\end{array}$ & $\begin{array}{l}\text { II: } 0.6 \mathrm{mg} \\
(n=6)\end{array}$ & & $\begin{array}{l}\text { III: } 1.5 \mathrm{mg} \\
(n=6)\end{array}$ & $\begin{array}{l}\text { IV: } 2.5 \mathrm{mg} \\
(n=6)\end{array}$ & & $\begin{array}{l}\mathrm{V}: 4.0 \mathrm{mg} \\
(n=3)\end{array}$ \\
\hline$t_{\max }(\mathrm{h})$ & $2.75(59)$ & $1.83(36)$ & & $0.92(56)$ & $1.58(83)$ & & $1.71(73)$ \\
\hline$C_{\max }(\mathrm{ng} / \mathrm{mL})$ & $1.11(24)$ & $3.21(12)$ & & $11.95(29)$ & $18.78(30)$ & & $38.05(33)$ \\
\hline $\mathrm{AUC}_{0-\mathrm{t}}(\mathrm{ng} \cdot \mathrm{h} / \mathrm{mL})$ & $28.03(12)$ & $83.32(13)$ & & $297.12(50)$ & $402.09(20)$ & & $550.01(17)$ \\
\hline $\begin{array}{l}\mathrm{AUC}_{0-\text { inf }}(\mathrm{ng} \cdot \mathrm{h} / \\
\mathrm{mL})\end{array}$ & $30.93(16)$ & 90.67 (13) & & $427.58(80)$ & $438.11(25)$ & & $568.46(18)$ \\
\hline $\mathrm{Vd} / \mathrm{F}(\mathrm{L})$ & $260(20)$ & $256(14)$ & & $213(9.7)$ & $203(9.5)$ & & 204 (17) \\
\hline $\mathrm{CL} / \mathrm{F}(\mathrm{L} / \mathrm{h})$ & $6.59(14)$ & $6.72(14)$ & & $5.34(52)$ & $6.03(26)$ & & $7.20(15)$ \\
\hline$t_{1 / 2}(\mathrm{~h})$ & $28(28)$ & $27(6.6)$ & & $41(76)$ & $25(28)$ & & $20(17)$ \\
\hline $\mathrm{MAD}^{\mathrm{a}}$ & $\begin{array}{l}\text { I: } 0.2 \mathrm{mg} / \text { day } \\
(n=6)\end{array}$ & $\begin{array}{l}\text { II: } 0.6 \mathrm{mg} / \mathrm{day} \\
(n=6)\end{array}$ & $\begin{array}{l}\text { III: } 1.0 \mathrm{mg} / \text { day } \\
(n=6)\end{array}$ & $\begin{array}{l}\text { IV: } 1.5 \mathrm{mg} / \text { day } \\
(n=6)\end{array}$ & $\begin{array}{l}\mathrm{V}: 2.5 \mathrm{mg} / \text { day } \\
(n=6)\end{array}$ & $\begin{array}{l}\text { Older adult: } 2.5 \\
\mathrm{mg} / \text { day }(n=6)\end{array}$ & $\begin{array}{l}\text { VI: } 4.0 \mathrm{mg} / \text { day } \\
(n=3)\end{array}$ \\
\hline $\begin{array}{l}t_{\max }, \text { ss (h after } \\
\text { last dosing) }\end{array}$ & $5.1(1.7)$ & $2.25(1.4)$ & $1.83(1.7)$ & $1.17(0.4)$ & $2.17(1.7)$ & $2.33(1.6)$ & $1.33(1.1)$ \\
\hline $\begin{array}{l}C_{\max }(\mathrm{ng} / \mathrm{mL}) \\
\text { (D1) }\end{array}$ & $1.28(23)$ & $3.69(22)$ & $6.83(56)$ & $11.35(30)$ & $26.64(54)$ & $24.28(12)$ & $55.97(27)$ \\
\hline$\underset{\text { (D5) }}{C_{\max , \mathrm{ss}}(\mathrm{ng} / \mathrm{mL})}$ & $2.32(17)$ & $7.65(39)$ & $14.11(43)$ & $23.61(18)$ & $49.92(59)$ & $41.00(42)$ & $109.73(50)$ \\
\hline $\begin{array}{l}\mathrm{AUC}_{0-t}(\mathrm{ng} \cdot \mathrm{h} / \mathrm{mL}) \\
\text { (D1) }\end{array}$ & $18.73(21)$ & $50.57(28)$ & $95.08(35)$ & $137.96(17)$ & $292.25(45)$ & $257.53(33)$ & $563.85(28)$ \\
\hline $\begin{array}{l}\mathrm{AUC}_{0-t, \mathrm{ss}}(\mathrm{ng} \cdot \mathrm{h} / \\
\mathrm{mL})(\mathrm{D} 5)\end{array}$ & $37.69(18)$ & $113.68(49)$ & $198.74(38)$ & $298.69(17)$ & $634.21(65)$ & $442.23(39)$ & $994.50(49)$ \\
\hline $\mathrm{RAC}$ & $1.91(17)$ & $1.90(22)$ & $1.92(9.2)$ & $1.87(7.0)$ & $1.86(7.2)$ & $1.92(6.7)$ & $1.79(15)$ \\
\hline$t_{1 / 2}(\mathrm{~h})$ & $22(25)$ & $22(33)$ & $23(14)$ & $22(11)$ & $22(11)$ & $23(10)$ & $20(23)$ \\
\hline $\operatorname{CSF}^{\mathrm{b}}$ & & & & & $\begin{array}{l}2.0 \mathrm{mg} \\
(n=3)\end{array}$ & & $\begin{array}{l}4.0 \mathrm{mg} \\
(n=3)\end{array}$ \\
\hline $\begin{array}{l}\mathrm{CSF}(\mathrm{pg} / \mathrm{mL}) \\
\text { @ } 2 \mathrm{~h}\end{array}$ & & & & & $239.42(56)$ & & $387.34(41)$ \\
\hline $\begin{array}{l}\mathrm{CSF}(\mathrm{pg} / \mathrm{mL}) \\
\text { @ } 6 \mathrm{~h}\end{array}$ & & & & & $181.47(11)$ & & 733.75 (27) \\
\hline $\begin{array}{l}\text { CSF (pg/mL) @ } \\
12 \mathrm{~h}\end{array}$ & & & & & $169.68(14)$ & & $549.93(5.3)$ \\
\hline $\begin{array}{l}\text { Plasma }_{\mathrm{u}}(\mathrm{pg} / \mathrm{mL}) \\
@ 2 \mathrm{~h}\end{array}$ & & & & & $315.68(56)$ & & $589.71(56)$ \\
\hline $\begin{array}{l}\operatorname{Plasma}_{\mathrm{u}}(\mathrm{pg} / \mathrm{mL}) \\
\text { @ } 6 \mathrm{~h}\end{array}$ & & & & & $248.31(17)$ & & $870.66(13)$ \\
\hline $\begin{array}{l}\text { Plasma }_{\mathrm{u}}(\mathrm{pg} / \mathrm{mL}) \\
@ 12 \mathrm{~h}\end{array}$ & & & & & $186.35(23)$ & & $609.11(12)$ \\
\hline $\begin{array}{l}\text { CSF/plasma } \\
\text { @ } 2 \text { h }\end{array}$ & & & & & $0.78(15)$ & & 0.69 (18) \\
\hline $\begin{array}{l}\text { CSF/plasma } \\
\text { @ 6h }\end{array}$ & & & & & $0.74(7.8)$ & & $0.83(14)$ \\
\hline $\begin{array}{l}\text { CSF/plasma } \\
12 \text { h }\end{array}$ & & & & & $0.92(8.9)$ & & $0.91(15)$ \\
\hline
\end{tabular}

Data are presented as mean (coefficient of variation, \%)

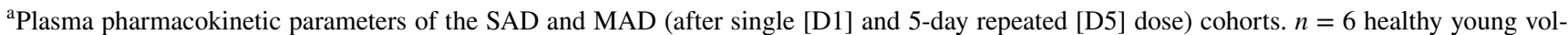
unteers per cohort (0.2-4 mg SAD and $0.2-2.5 \mathrm{mg}$ /day MAD), $n=3$ healthy young volunteers in the $4 \mathrm{mg} / \mathrm{day}$ MAD cohort, and $n=3$ older healthy adult volunteers in the $2.5 \mathrm{mg} /$ day cohort. MAD $\mathrm{AUC}_{\text {inf }}$ (D1) not reported as extrapolated AUC was $>20 \%$

${ }^{b}$ Pharmacokinetic parameters in the CSF cohort after administration of a single dose. CSF and plasma ${ }_{u}$ concentrations, and CSF-to-plasma $a_{u}$ ratios after a single dose of vafidemstat 2 or $4 \mathrm{mg} . n=3$ subjects per timepoint

$A U C_{0-i n f}$ area under the concentration-time curve within time 0 to infinity, $A U C_{0-t}$ area under the concentration-time curve within time $0-24$ h, $C L / F$ apparent clearance, $C_{\max }$ maximum (peak) concentration, $C S F$ cerebrospinal fluid, $D 1$ day $1, D 5$ day $5, M A D$ multiple ascending dose, plasma $_{u}$ plasma unbound, $R A C$ ratio of accumulation (calculated from $t_{1 / 2} / K_{\mathrm{e}} ; K_{e}$ elimination rate constant), $S A D$ single ascending dose, $s s$ steady state, $t_{1 / 2}$ terminal half-life, $t_{\max }$ time to reach $C_{\max }, V d / F$ apparent volume of distribution 


\section{Discussion}

Inhibitors of KDM1A have attracted attention for their potential use in the treatment of oncological diseases such as acute myeloid leukemia and small-cell lung cancer. Compounds currently undergoing clinical testing in oncology and hematology include iadademstat (ORY-1001) [18], bomedemstat (IMG-7289) [19], CC-90011 [20], and INCB059872 [21].

Vafidemstat (ORY-2001) is the first KDM1A inhibitor envisaged for CNS disease and evaluated in healthy volunteers. This study reports the results of a first-in-human phase I clinical trial of vafidemstat in healthy young and older adult volunteers to determine its safety and tolerability, to characterize its pharmacokinetics and pharmacodynamics, and to assess its CNS exposure. Vafidemstat was well-tolerated and did not produce any SAEs in the SAD or MAD studies. AEs were mild to moderate, and none were dose related, and the total frequency of AEs was similar in subjects receiving vafidemstat or placebo.

The pharmacokinetic data from this phase I trial indicate that vafidemstat is suitable for daily administration. The compound presents moderate accumulation after 5-day treatment, reaches steady state by day 5 , and is CNS penetrant. Whereas in vitro biochemical assays indicated near equipotency for KDM1A TE and MAOB inhibition, pharmacodynamic analyses on ex vivo samples from healthy volunteers revealed dose-dependent KDM1A TE in PBMCs but no platelet MAOB inhibition. These data correspond with previous ex vivo findings obtained in preclinical species that show vafidemstat acts primarily as a selective KDM1A inhibitor in vivo, with no significant pharmacological MAOB activity at the therapeutic doses used. No significant effects were detected on hematology in the SAD and MAD studies at the initially planned doses, and escalation in the MAD was continued until the expected hematological impact emerged. Platelet level was the only parameter that was significantly altered in this study, as expected and previously observed for other KDM1A inhibitors.

The obvious limitations of this first-in-human trial are the small number of subjects included in each dose level (especially in the older adult population), which may not reveal less frequent AEs, and the short treatment duration, which does not permit evaluation of long-term effects. Different phase IIa trials (see below) have indicated a good safety profile for vafidemstat even after long-term exposure (up to 18 months).

The dose range chosen in this clinical trial was the human equivalent of the dose range used in efficacy studies in rodent models. In rodents, vafidemstat showed good brain penetration and TE in brain and peripheric tissues and provided a 30-fold window between the therapeutic effect and hematopoietic impact. Importantly, in rodents, efficacy could be obtained at doses with no or negligible hematopoietic engagement. Based on the data obtained in this study and the characterization of platelet dynamics, phase II doses were established at 0.6 and $1.2 \mathrm{mg} /$ day. These doses were estimated to be sufficient to reach $\sim 60-80 \%$ KDM1A TE (efficacious in preclinical species) while avoiding a clinically relevant platelet impact, based on the exposure-response relationships (half and $80 \%$ maximal effective concentration). Evaluation of long-term data in phase IIa trials will permit vafidemstat dose selection for future pivotal phase III studies.

\section{Conclusion}

The 5-day treatment with vafidemstat was safe in the originally designed dose range. The pharmacodynamic results confirmed dose-dependent KDM1A TE but the absence of any significant MAOB inhibition at the doses used and confirmed the CNS penetration of vafidemstat. This phase I trial permitted the characterization of platelet dynamics and selection of phase IIa doses.

Vafidemstat has been tested in several phase IIa trials in patients with relapsing-remitting and secondary-progressive MS, in patients with mild to moderate Alzheimer's disease, and in patients displaying aggression in different psychiatric diseases and moderate to severe Alzheimer's disease (EUDRACT 2017-002838-23; 2017-004893-32; 2018002140-88; 2019-001436-54; NCT03867253). Based on the good safety profile in older adults in long-term treatments, and on the rapid and potent anti-inflammatory properties observed in models for MS, a randomized, open-label phase II study is ongoing to evaluate the ability of vafidemstat to prevent acute respiratory distress syndrome in severely ill adult patients with severe acute respiratory syndrome coronavirus 2 (SARS-CoV-2; coronavirus disease 2019) (EUDRACT N ${ }^{\circ}$ 2020-001618-39). Recent phase IIa efficacy data support vafidemstat as an emerging therapeutic option for treatment of agitation/aggression in psychiatric disorders such as attention-deficit/hyperactivity disorder, borderline personality disorder (BPD), autism spectrum disorder, and moderate-severe Alzheimer's disease [22, 23]. Based on these results, a multicenter, double-blind, randomized, placebo-controlled phase IIb trial to evaluate the efficacy and safety of vafidemstat in patients with BPD with agitation/ aggressiveness has been initiated (PORTICO; EUDRACT $\mathrm{N}^{\circ}$ 2020-003469-20), and an additional phase IIb trial to address cognitive impairment and negative symptoms in patients with schizophrenia is planned to launch in 2021. 
a

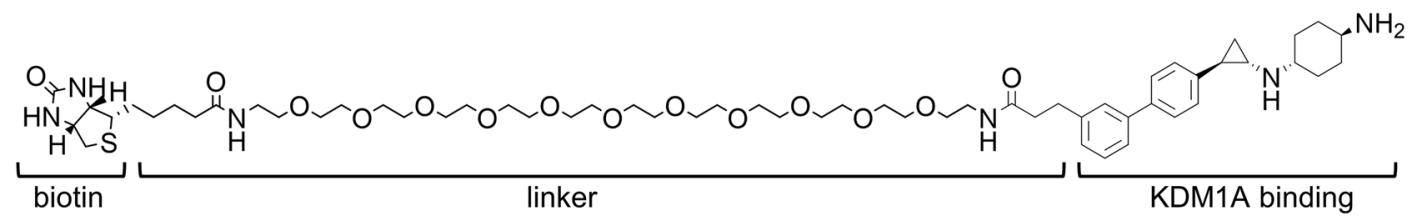

b

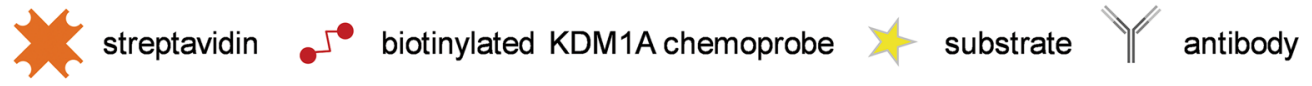

Blood

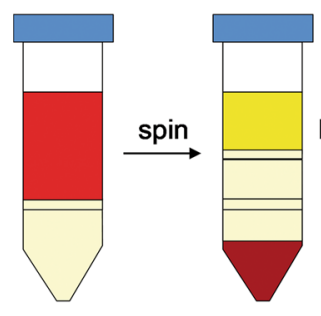

PBMCs

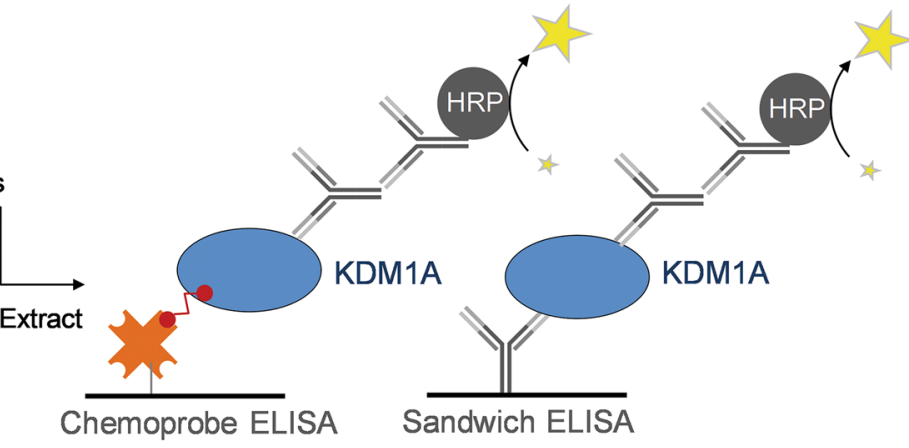

C

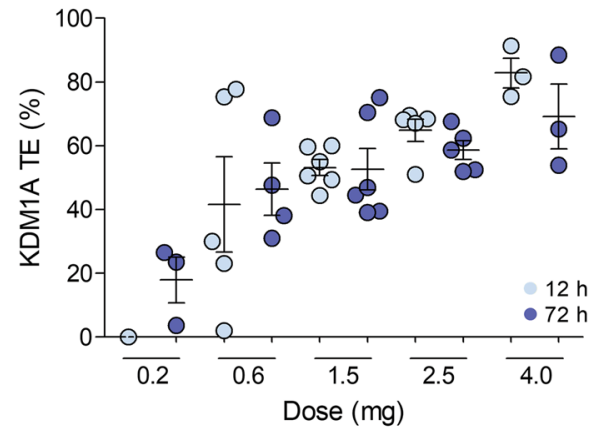

e

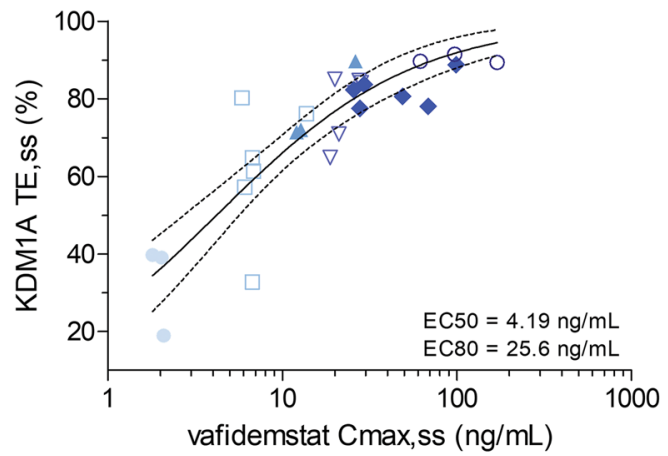

g

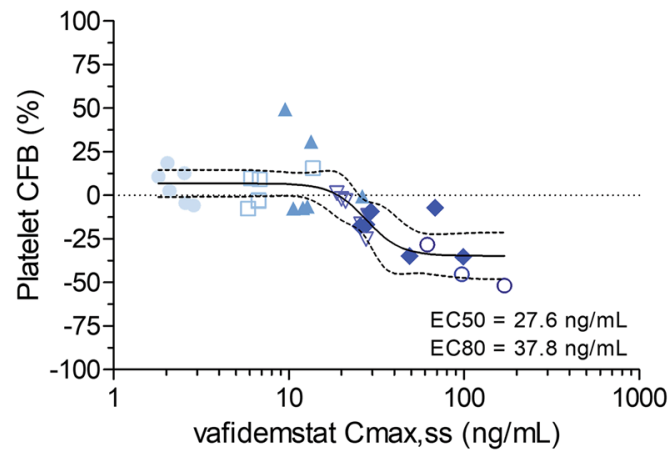

d

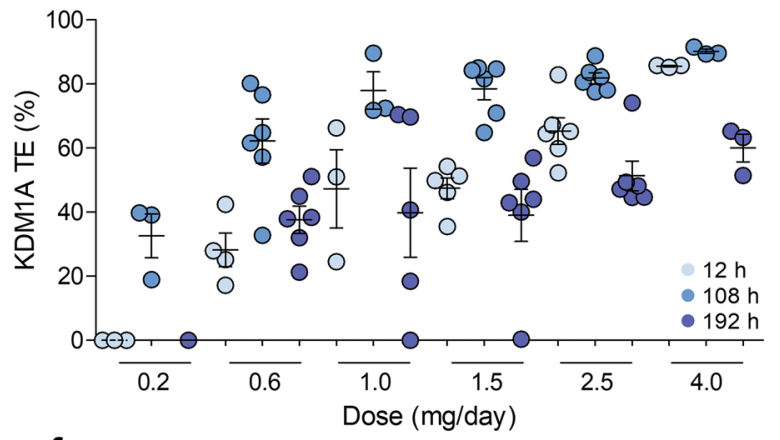

f

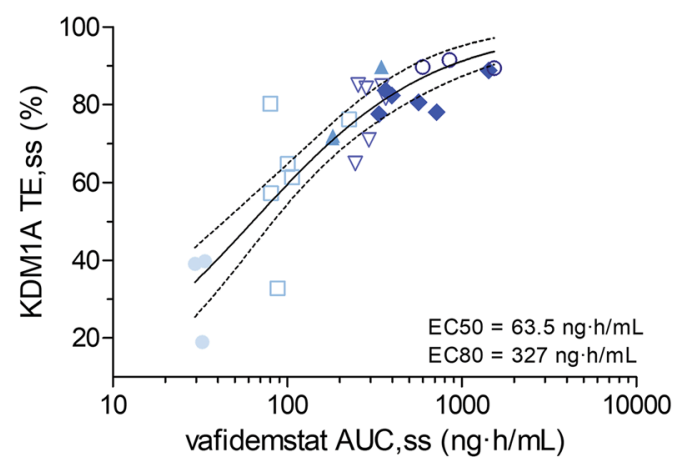

h

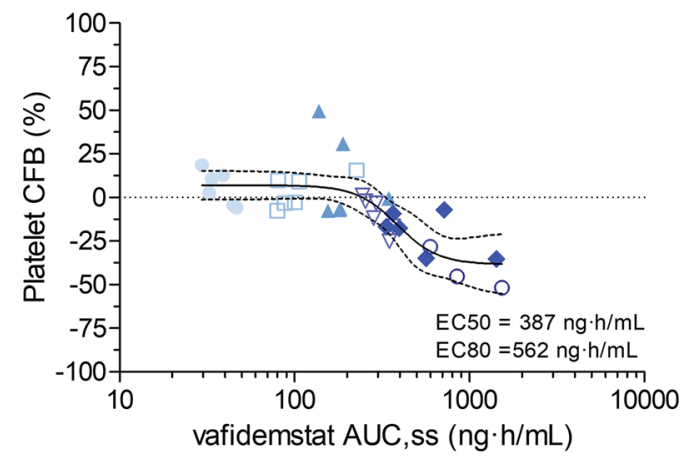


४Fig. 4 Vafidemstat pharmacodynamics. a The KDM1A chemoprobe OG-881 used to capture uninhibited protein. b Graphical representation of the chemoprobe and sandwich ELISA used to determine free and total KDM1A. c KDM1A TE after SAD and d MAD in healthy young subjects. Light blue dots $12 \mathrm{~h}$; medium blue dots $108 \mathrm{~h}$; dark blue dots $72 \mathrm{~h}$ (SAD) or $192 \mathrm{~h}$ (MAD) after the first dose. Data are presented as mean and standard error of the mean. Volunteers sampled at each dose: $n=6$ except MAD $4 \mathrm{mg}, n=3$. e, f Pharmacokinetic/pharmacodynamic relationship between exposure, as reflected by $C_{\text {max,ss }}$ or AUCss, and KDM1A TE. g, h Pharmacokinetic/pharmacodynamic relationship between exposure, as reflected by $C_{\max , \mathrm{ss}}$ or AUCss, and platelet dynamics (represented as \% CFB levels). All values correspond to independent subjects. Calculated $\mathrm{EC}_{50} / \mathrm{EC}_{80}$ values are also shown in the respective graphs. Light blue circles 0.2 $\mathrm{mg}$; white squares $0.6 \mathrm{mg}$; light blue triangles $1 \mathrm{mg}$; white triangles $1.5 \mathrm{mg}$; blue diamonds $2.5 \mathrm{mg}$; white circles $4 \mathrm{mg}$; full lines nonlinear fit; dotted lines $95 \%$ confidence interval. Volunteers sampled at each dose: $n=6$ except MAD $4 \mathrm{mg}, n=3$. Samples that did not pass quality control were omitted. $A U C$, ss area under the plasma concentration-time curve at steady state, $C F B$ change from baseline, $C_{\text {max }, s s}$ maximum concentration at steady state, $E C_{50}$ half maximal effective concentration, $E C_{80} 80 \%$ maximal effective concentration, ELISA enzyme-linked immunosorbent assay, $H R P$ horseradish peroxidase, $M A D$ multiple ascending dose, $P B M C s$ peripheral blood mononuclear cells, $S A D$ single ascending dose, $S S$ steady state, $T E$ target engagement

Supplementary Information The online version contains supplementary material available at https://doi.org/10.1007/s40263-021-00797-x.

Acknowledgements The authors are grateful to the volunteers who participated in this clinical trial.

\section{Declarations}

Author contributions Conceptualization: RMA, TM, CMo, CB. Formal analysis: MIA, RMA, JMF-C, JM-C, JC. Funding acquisition: CB, TM. Investigation: RMA, JMF-C, JMC, JC, CMa, MIA. Methodology: TM. Project Administration: CMo, RMA. Resources: RMA, JMF-C, JM-C, JC. Supervision: RMA, CMo. Visualization: MIA. Original draft preparation: RMA, TM, MIA. All authors contributed to the review and editing of this article. All authors approved the content of the manuscript and take responsibility for the content of the article.

Funding The open access fee was paid by Oryzon Genomics, S.A. This study was funded by Oryzon Genomics, S.A. (the sponsor), and grant CIIP-20152001/E9683, EUROSTARS-2/CDTI, Spanish Ministry of Science, Innovation and Universities.

Conflict of interest RMA has received personal fees from Centre d'Investigació del Medicament, Institut de Recerca de l'Hospital de la Santa Creu@ Sant Pau (IIB-Sant Pau) and Hospital de la Santa Creu i Sant Pau, Barcelona, Spain. JMF-C, JC, MP, and JM-C have received personal fees from Centre d'Investigació del Medicament, Institut de Recerca de l'Hospital de la Santa Creu i Sant Pau (IIB-Sant Pau), Barcelona, Spain. MIA, CMa, CMo, CB, and TM have received personal fees from Oryzon Genomics, during the conduct of the study. In addition, CMa is listed as inventor on patent application WO2017/158136 (pending), assigned to Oryzon Genomics; CMo owns company shares on the stock exchange (Oryzon Genomics); and CB reports patent WO2012/013728 (issued in multiple countries), assigned to Oryzon Genomics. CB is executive director and shareholder of Oryzon Genom- ics. TM has received other compensation from the Alzheimer's Drug Discovery Foundation, outside the submitted work; is listed as inventor in WO2017/158136 (pending), WO2017/212061 (issued in multiple countries), and WO2019/025588 (pending) and further reports patent WO2012/013728 (issued in multiple countries), all assigned to Oryzon Genomics; is executive director and shareholder of Oryzon Genomics; and is a member of the Scientific Review Board of the Alzheimer's Drug Discovery Foundation, for which no compensation is received.

Ethics approval This study was registered under EUDRACT No. 2015-003721-33 and carried out in accordance with the protocol CL01-ORY-2001 and good clinical practices (Declaration of Helsinki, guideline ICH CPMP/ICH/135/95, European Directive 2001/20/EC, and Spanish RD 561/1993 and RD 223/2004). The study protocol and informed consent forms were approved by the Spanish Agency for Medicines and Health Products and the institutional review board.

Consent to participate Written informed consent was obtained from all candidate subjects participating in this study before any clinical procedures were undertaken.

Consent for publication The written informed consent allows publication of the data included in this manuscript.

Availability of data and material Materials can be obtained through a material transfer agreement upon reasonable request. The KDM1A Target Engagement Method validation protocol and report are available upon request. Access to the full trial protocol and clinical trial data can be obtained through a data-sharing agreement upon reasonable request and in compliance with applicable laws. Contact the corresponding author or emailinfo@oryzon.com.

Code availability Not applicable.

Open Access This article is licensed under a Creative Commons Attribution-NonCommercial 4.0 International License, which permits any non-commercial use, sharing, adaptation, distribution and reproduction in any medium or format, as long as you give appropriate credit to the original author(s) and the source, provide a link to the Creative Commons licence, and indicate if changes were made. The images or other third party material in this article are included in the article's Creative Commons licence, unless indicated otherwise in a credit line to the material. If material is not included in the article's Creative Commons licence and your intended use is not permitted by statutory regulation or exceeds the permitted use, you will need to obtain permission directly from the copyright holder. To view a copy of this licence, visit http://creativecommons.org/licenses/by-nc/4.0/.

\section{References}

1. Barter JD, Foster TC. Aging in the brain: new roles of epigenetics in cognitive decline. Neuroscientist. 2018;24(5):516-25.

2. Wood IC. The contribution and therapeutic potential of epigenetic modifications in Alzheime's disease. Front Neurosci. 2018;12:649.

3. Collins BE, Greer CB, Coleman BC. Histone H3 lysine K4 methylation and its role in learning and memory. Epigenet Chromatin. 2019;12(1):7.

4. Lewis CR, Candace R, Lewis CR, Henderson-Smith A, Reagan S, Breitenstein RS, et al. Dopaminergic gene methylation is associated with cognitive performance in a childhood monozygotic twin study. Epigenetics. 2019;14(3):310-23. 
5. Delgado-Morales R, Agís-Balboa RC, Esteller M. Epigenetic mechanisms during ageing and neurogenesis as novel therapeutic avenues in human brain disorders. Clin Epigenet. 2017;9:67.

6. Maes T, Mascaró C, Ortega A, Lunardi S, Ciceri F, Somervaille $\mathrm{TCP}$, et al. KDM1 histone lysine demethylases as targets for treatments of oncological and neurodegenerative disease. Epigenomics. 2015;7(4):609-26.

7. Matsuda S, Baba R, Oki H, Morimoto S, Toyofuku M, Igaki S, et al. T-448, a specific inhibitor of LSD1 enzyme activity, improves learning function without causing thrombocytopenia in mice. Neuropsychopharmacology. 2019;44(8):1505-12.

8. Mukai J, Cannavò E, Crabtree GW, Sun Z, Diamantopoulou A, Thakur P, et al. Recapitulation and reversal of schizophrenia-related phenotypes in Setd1a-deficient mice. Neuron. 2019;104(3):471-87.e12.

9. Shi Y, Lan F, Matson C, Mulligan P, Whetstine JR, Cole PA, et al. Histone demethylation mediated by the nuclear amine oxidase homolog LSD1. Cell. 2004;119(7):941-53.

10. Maes T, Mascaró C, Rotllant D, Lufino MMP, Estiarte A, Guibourt N, et al. Modulation of KDM1A with vafidemstat rescues memory deficit and behavioral alterations. PLoS ONE. 2020;15(5):e0233468.

11. Maes T, Cavalcanti F, González-Rey E, Delgado M, Mascaró C, Lufino MMP, et al. Characterization of the efficacy of ORY-2001, a novel epigenetic drug for the treatment of multiple sclerosis, during the effector phase of the EAE model [Abstract]. ECTRIMS Online Library. Maes T. 10/26/17; 200311; P656.

12. Saleque S, Kim J, Rooke HM. Epigenetic regulation of hematopoietic differentiation by Gfi-1 and Gfi- $1 \mathrm{~b}$ is mediated by the cofactors CoREST and LSD1. Mol Cell. 2007;27(4):562-72.

13. Sprüssel A, Schulte JH, Weber S, Necke M, Händschke K, Thor $\mathrm{T}$, et al. Lysine-specific demethylase 1 restricts hematopoietic progenitor proliferation and is essential for terminal differentiation. Leukemia. 2012;26(9):2039-51.

14. EMA. European Medicines Agency. Guideline on requirements for first-in-man clinical trials for potential High-Risk medicinal products. 2007. EMEA/CHMP/SWP/28367/2007Corr.

15. FDA. U.S. Food and Drug Administration: Guidance for industry: estimating the maximum safe starting dose in initial clinical trials for therapeutics in adult healthy volunteers. 2005.
16. Mascaró C, Ortega A, Carceller E, Ruiz Rodriguez R, Ciceri F, Lunardi S, et al. Chemoprobe-based assays of histone lysine demethylase $1 \mathrm{~A}$ target occupation enable in vivo pharmacokinetics and pharmacodynamics studies of KDM1A inhibitors. J Biol Chem. 2019;294(20):8311-22.

17. Mascaró C, Ruiz Rodriguez R, Maes T. Direct measurement of KDM1A target engagement using chemoprobe-based immunoassays. J Vis Exp. 2019. https://doi.org/10.3791/59390.

18. Salamero O, Montesinos P, Willekens C, Pérez-Simón JA, Pigneux A, Récher C, et al. First-in-human phase I study of Iadademstat (ORY-1001): a first-in-class lysine-specific histone demethylase 1A inhibitor, in relapsed or refractory acute myeloid leukemia. J Clin Oncol. 2020;38(36):4260-73.

19. Pettit K, Gerds AT, Tacoub A, Watts JM, Tartaczuch M, Bradley TJ, et al. A Phase 2a Study of the LSD1 Inhibitor Img-7289 (bomedemstat) for the treatment of myelofibrosis [Abstract]. Blood. 2019;134(Supplement_1):556.

20. Hollebecque A, de Bono JS, Plummer R, Isambert N, Martin-Romano P, Baudin E, et al. Phase I study of CC-90011 in patients with advanced solid tumors and relapsed/refractory nonHodgkin lymphoma (R/R NHL) [Abstract]. 2018. Ann Oncol. 2018;29(suppl_8):viii649-viii669.

21. Chadderton A, Ye M, Diamond M, Roman V, Weber M, He C, et al. The FAD-directed LSD1 specific inhibitor, INCB059872, is a promising epigenetic agent for AML therapy by inducing differentiation of leukemic stem/progenitor cells [Abstract]. Cancer Res. 2018;78(13 Suppl):1888.

22. Ramos-Quiroga JA, Bullock R, Ferrer M, Fadeuilhe C, Gutiérrez S, Richarte V, et al. PS01.48. Vafidemstat: an epigenetic drug with emerging therapeutic potential, composite data from three psychiatric disorders from the REIMAGINE trial [Abstract]. 2019 CINP Intl. Meeting, Athens, 3-5 October 2019. http://bit.ly/2Dxmtj0. http://bit.ly/2DzW4RL. Accessed 22 Feb 2021.

23. Bullock R, Ferrer M, Gutierrez S, Richarte V, Gisbert L, Lara B, et al. EPP0681. Vafidemstat reduces aggressiveness in three different psychiatric disorders. Final data from the reimagine trial. [Abstract]. European Psychiatry, Volume 63, Special Issue S1: Abstracts of the 28th European Congress of Psychiatry, 2020, S170-S171. https://doi.org/10.1192/j.eurpsy.2020.5. 